\title{
Study of Bifocal Dual Reflectarray Configurations for Multi-Beam Antennas in Ka-Band
}

\author{
Eduardo Martinez-de-Rioja, Jose A. Encinar
}

\author{
Ciaran Geaney, Sean V. Hum
}

\author{
Antonio Pino
}

\begin{abstract}
This paper describes the design of multi-beam dual reflectarray antennas for operation in the Ka-band satellite transmission band (around $20 \mathrm{GHz}$ ), using the bifocal design concept to improve antenna performance. This technique has been first applied to obtain beam compression with adjacent feeds, resulting in low radiation efficiency. Then it has been used without beam compression to correct beam aberration, showing better results than an equivalent single-focus reference reflector. The simulated radiation patterns in the elevation plane and the amplitude distributions of the incident field on each reflectarray have been calculated for different antenna configurations.
\end{abstract}

\section{INTRODUCTION}

The design of multi-beam antennas for telecommunication satellites in Ka-band must deal with challenging requirements, such as the generation of a large number of high-gain spot beams, close separation between the beams, and alternation of frequencies and/or polarizations for adjacent beams [1]. In this paper, the capability of dual reflectarray antennas to generate multiple beams in Ka-band is studied, using the bifocal design concept to obtain closer beams or improve the performance of the multi-beam antenna.

\section{BIFOCAL TECHNIQUE APPLIED TO REFLECTARRAYS}

The classic bifocal synthesis technique for dual offset reflector configurations is a way to improve the scanning performance of reflector antennas [2]. This method considers two focal points, $F_{1}$ and $F_{2}$, which generate two beams in the directions $\theta_{\mathrm{b} 1}$ and $\theta_{\mathrm{b} 2}$ by shaping both reflectors. The bifocal technique was recently applied to centered dual reflectarray configurations of small size [3] [4]. The main differences with respect to reflector antennas are the use of planar instead of curved surfaces, and the substitution of normal vectors with the phase derivative at each reflectarray point [3]. The incidence $\left(\theta_{i}\right)$ and reflection $\left(\theta_{0}\right)$ angles of the rays on each reflectarray cell are related to the phase derivative $(d \Phi / d r)$ by:

$$
\frac{2 \pi}{\lambda} \cdot\left(\sin \theta_{i}-\sin \theta_{o}\right)=\frac{d \Phi}{d r}
$$

The integration of the interpolated phase derivative curves provides the phase profile on each reflectarray. If the initial conditions of the bifocal synthesis exhibit axial symmetry (see
Fig. 1(a)), the two phase curves can be rotated around the symmetry axis to obtain a surface phase distribution for each reflectarray, producing a focal ring that contains $\mathrm{F}_{1}$ and $\mathrm{F}_{2}[5]$.

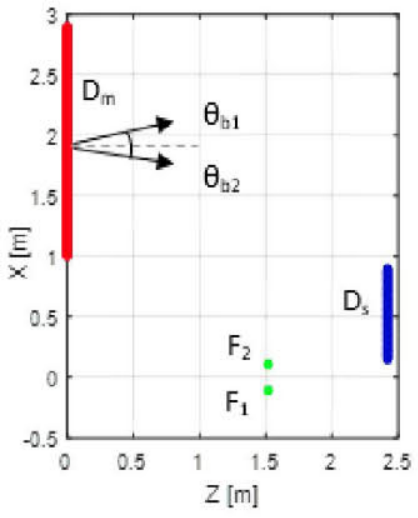

(a)

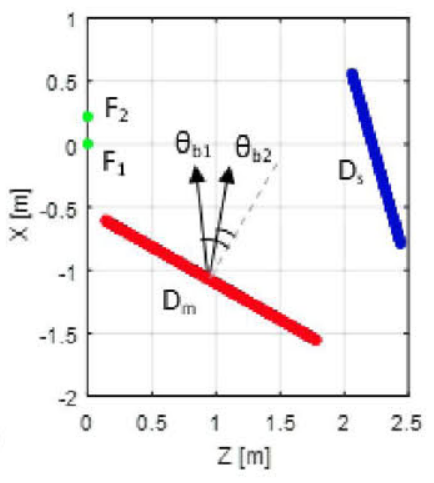

(b)
Fig. 1. Geometry of the bifocal dual reflectarray antenna: (a) Cassegrain configuration, (b) compact-range configuration.

\section{BIFOCAL TECHNIQUE TO OBTAIN CLOSER BEAMS}

The bifocal design method has been applied to different dual reflectarray configurations in order to obtain beam compression, meaning that the separation between beams generated with adjacent feeds is so small that it would not be possible with a single-focus antenna, as it would require overlapping feeds. For this purpose, $54 \mathrm{~mm}$ diameter feedhorns were considered in order to produce a taper level of -12 $\mathrm{dB}$ at the reflector edge when illuminating with $36^{\circ}$ subtended

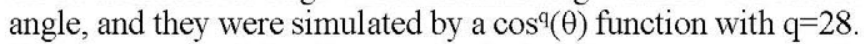
The geometry of the Cassegrain dual reflectarray antenna is shown in Fig. 1(a). The diameters of the main and sub reflectarrays are $D_{m}=1.9 \mathrm{~m}$ and $D_{s}=80 \mathrm{~cm}$, in order to ensure around $50 \mathrm{~dB}$ of gain. The separation between the foci of the bifocal synthesis is $21.6 \mathrm{~cm}$, considering a linear array of 5 nonoverlapping feeds, where the phase centers of the first and the fifth feeds would be placed at $F_{1}$ and $F_{2}$, respectively. The design beam directions are $\theta_{\mathrm{b} 1}=1.12^{\circ}$ and $\theta_{\mathrm{b} 2}=-1.12^{\circ}$ in order to obtain $0.56^{\circ}$ separation between adjacent beams, which would traditionally require $50 \%$ feed overlap. After applying 
the bifocal technique, the simulated radiation patterns of the antenna at $20 \mathrm{GHz}$ assuming ideal elements have been calculated (see Fig. 2).

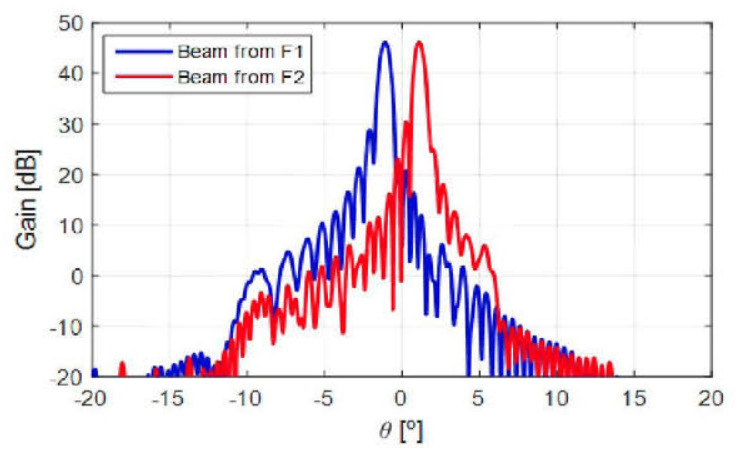

Fig. 2. Simulated radiation patterns in the elevation plane.

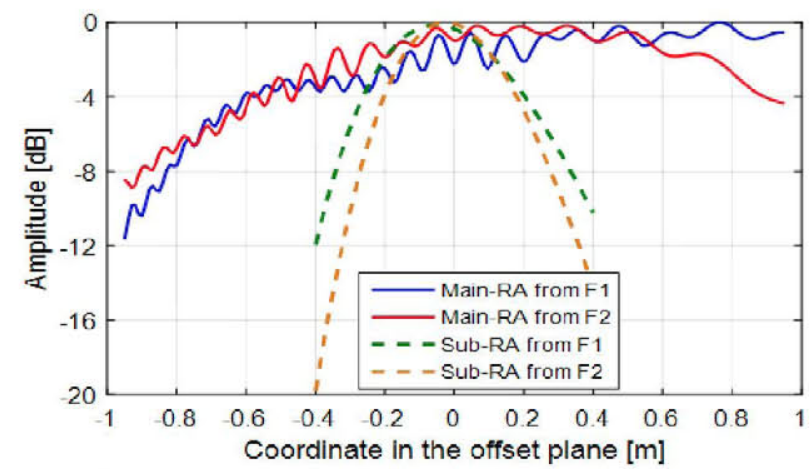

Fig. 3. Amplitude of the incident field on both reflectarrays.

Despite achieving the required beam compression, the antenna presents a serious efficiency problem, which can be noticed in the reduced gain. The amplitude distributions of the incident field over the symmetry axis of both reflectarrays for illumination from $F_{1}$ and $F_{2}$ are shown in Fig. 3. Note that the levels of illumination on the edges of the main reflectarray are very high. Introducing some modifications in the initial conditions of the bifocal synthesis or changing the antenna configuration (Gregorian, compact-range) does not mitigate this problem. The only possible solution would be to increase the size of the main reflectarray, but this implies using a very large reflectarray around $3.8 \mathrm{~m}$ in diameter.

\section{BIFOCAL TECHNIQUE TO CORRECT BEAM ABERRATION}

The bifocal algorithm has been used to correct beam aberration and improve the multi-beam performance of the dual reflectarray antenna, with no beam compression. In this case, the bifocal synthesis has been applied to a compact-range geometry, shown in Fig 1(b). The separation between the foci is again 21.6 $\mathrm{cm}$, and the diameters of the two reflectarrays are $\mathrm{D}_{\mathrm{m}}=1.9 \mathrm{~m}$ and $D_{\mathrm{s}}=1.4 \mathrm{~m}$. The separation between the beams is now $1.12^{\circ}$ instead of $0.56^{\circ}$, so $\theta_{\mathrm{b} 1}=28.24^{\circ}$ and $\theta_{\mathrm{b} 2}=23.76^{\circ}$. The new requirements produce reasonable illumination tapers on both reflectarrays, increasing the radiation efficiency (see Fig. 4). The compact range geometry forces the use of a larger subreflectarray, although proper illumination is obtained for all the feeds. The comparison of the beams generated by the bifocal antenna and those produced by a single focus reference reflector is shown in Fig. 5. There is a significant improvement in the single-entry $\mathrm{C} / \mathrm{I}$ ratio (where the interference is produced by the radiation of the adjacent beams), as can be inferred from the lower side-lobe levels.

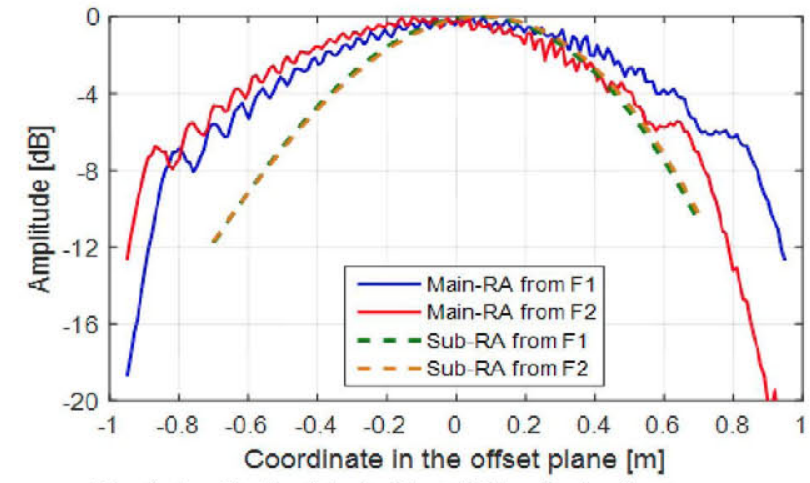

Fig. 4. Amplitude of the incident field on both reflectarrays.

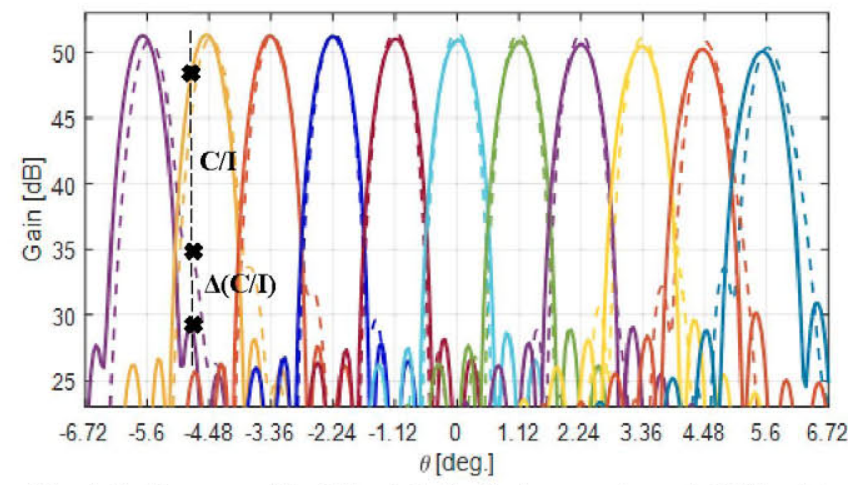

Fig. 5. Performance of the bifocal dual reflectarray antenna (solid lines) in comparison with the single-focused reference reflector (dashed lines).

\section{CONCLUSIONS}

The results show the efficiency problem when the bifocal technique is used to compress the beams. However, it seems to work best when is only used to correct beam aberration. The simulated radiation patterns are very promising for multi-spot beam antennas in Ka-band.

\section{ACKNOWLEDGMENT}

This work has been supported by the Spanish Ministry of Economy and Competitiveness under the projects TEC201343345-P, TEC2015-65353-R and TEC2016-75103-C2-1-R, by the Galician Regional Government (Project GRC2015/18), and by the European Space Agency (ESA) under contract $4000117113 / 16 / \mathrm{NL} / \mathrm{AF}$.

\section{REFERENCES}

[1] M. Schneider, C. Hartwanger, H. Wolf, "Antennas for multiple spot beams satellites", CEAS Space Journal, Vol. 2, pp. 59-66, Dec. 2011.

[2] C. M. Rappaport, "An offset bifocal reflector antenna design for wideangle beam scanning", IEEE Trans. on Antennas \& Propag., Vol. 32, No. 11, pp. 1196-1204, Nov. 1984.

[3] W. Menzel, M. Al-Tikriti, R. Leberer, "A 76-GHz multiple-beam planar reflector antenna", Proc. $32^{\text {nd }}$ Eur. Microw. Conf., Milan, Italy, 2002.

[4] J. G. Cuevas et al, "Principle of bifocal antennas implemented in a dual reflectarray configuration", $9^{\text {th }}$ EuCAP, Lisbon, Portugal, April 2015.

[5] E. Martinez-de-Rioja et al, "Application of Bifocal Concept to Dual Reflectarray Configurations for Multi-Beam Dual Reflectarray Antennas in Ka-Band", accepted at 11 ${ }^{\text {th }}$ EuCAP, Paris, France, March 2017. 